\title{
Lithography-free and Highly Angle Sensitive Structural Coloration Using Fabry-Perot Resonance of Tin
}

\author{
Young-Gyun $\mathrm{Kim}^{1} \cdot$ Ying-Jun Quan ${ }^{2} \cdot$ Min-Soo Kim ${ }^{1} \cdot$ Younggyun $\mathrm{Cho}^{1} \cdot$ Sung-Hoon Ahn ${ }^{1,2}$ (])
}

Received: 17 October 2020 / Revised: 29 January 2021 / Accepted: 9 February 2021 / Published online: 22 March 2021

(c) The Author(s) 2021

\begin{abstract}
Recently, there has been much interest in applying the color changes of nano-patterned structures to sensor technology. However, the lithographic nano-patterning process is not environmentally friendly, and it is difficult to fabricate large areas of color due to limitations associated with this approach. In this study, we realized a highly tunable structural coloration based on a Fabry-Perot interferometer design that does not require nano-patterning processes. To increase the reflected color change according to the angle, a color element using an asymmetric metal-insulator-metal structure was applied, fabricated using silver-silicon dioxide-tin ( $\mathrm{Sn}$ ), respectively. Using the optical properties of Sn, we maximized the change in reflection color and realized three primary colors of subtractive color of cyan, magenta and yellow according to the angle of designed MIM structure. Theoretical and experimental results revealed that the color and intensity of the reflectance depended strongly on the angle of the reflective surfaces. The manufacturing process is simple and yields large surfaces of high quality. Based on this premise, we fabricated a soft robot capable of color camouflage, and an angle-detecting color sensor for inspecting the three-dimensional shape quality of curved glass with a high sensitivity of $1.8 \mathrm{~nm} /$ degree. In addition, we propose a shape evaluation method by color, spectrometry, and monochromatic light.
\end{abstract}

Keywords Lithography-free $\cdot$ Metamaterials $\cdot$ Thin films $\cdot$ Plasmonics $\cdot$ Metal-insulator-metal

\section{Introduction}

The use of micro-nanotechnologies to obtain structural coloration has gained much attention recently. In particular, the technology of color variation as an indication of structural features and physical mechanisms has been actively researched for applications involving display devices [1,2], sensors [3, 4], and soft biosensors [5] for use in research, manufacturing, and smart factories. In contrast to conventional color technology that uses pigments or dye to color items via industrial processes, structural coloration is based on nano-patterns or multiple nano-film layers to generate color with light reflection. Because it does not

Sung-Hoon Ahn

ahnsh@snu.ac.kr

1 Department of Mechanical Engineering, Seoul National University, 1 Gwanak-ro, Gwanak-gu, Seoul 08826, Republic of Korea

2 Institute of Advanced Machines and Design, Seoul National University, 1 Gwanak-ro, Gwanak-gu, Seoul 08826, Republic of Korea induce chemical reactions or changes in material composition, structural coloration has many advantages, including brightness, clarity, and sustainability. Structural color is an example of nanoparticle technology resulting from the periodicity introduced by surface plasmon resonance [6-8] and photonic crystals [9-13]. In the case of surface plasmon resonance, color is expressed by free electrons resonating on the surface of a metal conductor in response to incident light [14]. A Fabry-Perot (FP) interferometer constructed with nanoscale thin films of metals and insulating layers can be used effectively to create large-area nanoscale structures via the surface plasmon effect in the metal [15]. The FP interferometer is a light-emitting device that uses a reflection system consisting of two thin parallel mirrors to repeatedly reflect light, thus causing interference and resonant light emission. This type of interferometer usually has a metal-insulator-metal (MIM) structure, in which the metal layers act as the reflective surfaces and the intermediate insulating layer constitutes the resonance layer $[16,17]$. A method for realizing structural coloration, i.e., by stacking two-dimensional nanostructures, has already been commercialized and enables precise control of thin-film thickness through physical 
vapor deposition processes $[18,19]$. Due to its simple fabrication and good performance, various studies are underway to expand the applications of structural colors realized using an FP interferometer [20-26]. FP color has an angle dependence, which can be a disadvantage in some applications involving etalons and color filters [27-30]. However, in sensing applications involving reflected light, the resulting structural coloration according to the angle of the reflected surface can be useful.

In this study, we demonstrated a highly angle sensitive and broadband structural coloration using an asymmetric MIM structure.

To generate angle-sensitive vivid structural colors, a silver $(\mathrm{Ag})$-silicon dioxide $\left(\mathrm{SiO}_{2}\right)$-tin ( $\left.\mathrm{Sn}\right)$ MIM structure was fabricated using an electron-beam evaporation process. We experimentally showed the structural color changes with different reflected angles. The results were verified with finite-difference time domain (FDTD) simulation results. As for the fabricated structural color, the central wavelength of absorption changed linearly and greatly, and the color change according to the angle was expressed as a function of reflected angle to spectra. Finally, a highly angle sensitive structural coloration is demonstrated using pattern-free, lithography free manufacturing process.

\section{Results and Discussion}

The main purpose of this research was to design a tunable structural colors that is highly sensitive to reflected angle. We used an asymmetric MIM structure to create the structural colors of an FP interferometer [31]. In nature, a butterflies camouflage themselves by changing the color of wings. Figure 1a shows the butterfly changing the color of its wings similar to surrounding color. Figure $1 \mathrm{~b}$ presents the MIM structure composed of $\mathrm{Ag}-\mathrm{SiO}_{2}-\mathrm{Sn}$ layers and the angledependent color change; the color changed significantly with the angle due to the MIM asymmetry and the optical properties of Sn. In the MIM structure, the top and bottom surfaces are metals, with a transparent insulating layer in between. For white light incident on the structure, the FP interferometer generates visible color through light resonance in the insulator layer; the color depends on the angle, as shown in Fig. 1b. We fabricated a butterfly robot capable of color camouflage operated by a spring made of shape memory alloy (SMA) with a phase transition temperature of $40^{\circ}$, as shown in Fig. 1c (Figure S1, Supporting Information). The realized colors of the wing covered all subtractive colors including cyan, magenta and yellow. Hence, as shown in Fig. 1d, the embodied structural colors enable the camouflage of butterfly into surrounding environment.

Figure 2a presents a schematic diagram of the MIM structure that induces the FP effect and the structural coloration.
The phase of the wavelength of the internal light varies with the insulator thickness. The reflectance of light (R) in a cavity can be expressed as follows [32]:

$R=\frac{4 r \sin ^{2} \delta}{(1-r)^{2}+4 r \sin ^{2} \delta}$

where $\mathrm{r}$ is the reflectance between the medium and the cavity, $\delta(\delta=2 \pi n d / \lambda)$ is the phase difference, $n$ is the refractive index, $d$ is the cavity length, and $\lambda$ is the wavelength of incident light. When the white incident light illuminates the MIM structure, light passes through the upper metal layer to the other layers. The interference of light between $\mathrm{Sn}$ and Ag layers separated by the $\mathrm{SiO}_{2}$ layer generates a resonance peak; thus, color is generated on the top surface, in this case, $\mathrm{Sn}$. The bottom layer of the MIM structure was Ag, which has high reflectivity [13]. The middle layer, silicon dioxide $\left(\mathrm{SiO}_{2}\right)$, is a low-cost, easily manufactured, and commercially viable material [33]. The top layer of the MIM structure was fabricated with $\mathrm{Sn}$, which is highly reflective and selectively refracts light of a specific wavelength. The light absorption properties of Sn create a MIM structure with a wide absorption bandwidth in the visible light region, providing a full, vivid color spectrum, even with reflected light. Sn is widely used in industry because it can be deposited at a relatively low temperature and yield uniform film quality. Furthermore, it has good radio wave permeability compared with other industrial metals such as aluminum, making it particularly well suited for applications in electronic devices such as mobile phones [34].

In the proposed MIM structure shown in Fig. 2a, external light is incident on the upper Sn layer. The thickness of the Sn layer was set to $10 \mathrm{~nm}$ by considering the effect of the asymmetry of the MIM structure on the angle dependence of the color change and the visibility of color [35]. Resonant light, which has a specific wavelength, exits to the outside; thus, the refractive index of the upper metal layer plays an important role in color generation. In the visible light region, the refractive index of Sn varies significantly by wavelength. To obtain the refractive index of Sn, we fabricated a 500-nm-thick Sn bulk coating sample and measured its refractive index using ellipsometry. The refractive index of Sn exhibited an increasing trend (from $n=2.0-4.3$ ) in the visible light region from $400-800 \mathrm{~nm}$ (Fig. 2b). Figure 2c presents the simulation results of the reflectance value of the $\mathrm{Ag}(100 \mathrm{~nm})-\mathrm{SiO}_{2}(0 \sim 500 \mathrm{~nm})-\mathrm{Sn}(10 \mathrm{~nm})$ structure using FDTD analysis based on the refractive index data presented in Fig. 2b. Compared with the $\mathrm{Ag}-\mathrm{SiO}_{2}-\mathrm{Ag}$ structure (Figure $\mathrm{S} 2$, Supporting Information), the $\mathrm{Ag}-\mathrm{SiO}_{2}-\mathrm{Sn}$ sample absorbed over a wider wavelength range, as shown in Fig. 2c. In the MIM structure in which the resonance light is absorbed, it is difficult to visually recognize color change due to the high-gloss surface; this occurs when the 

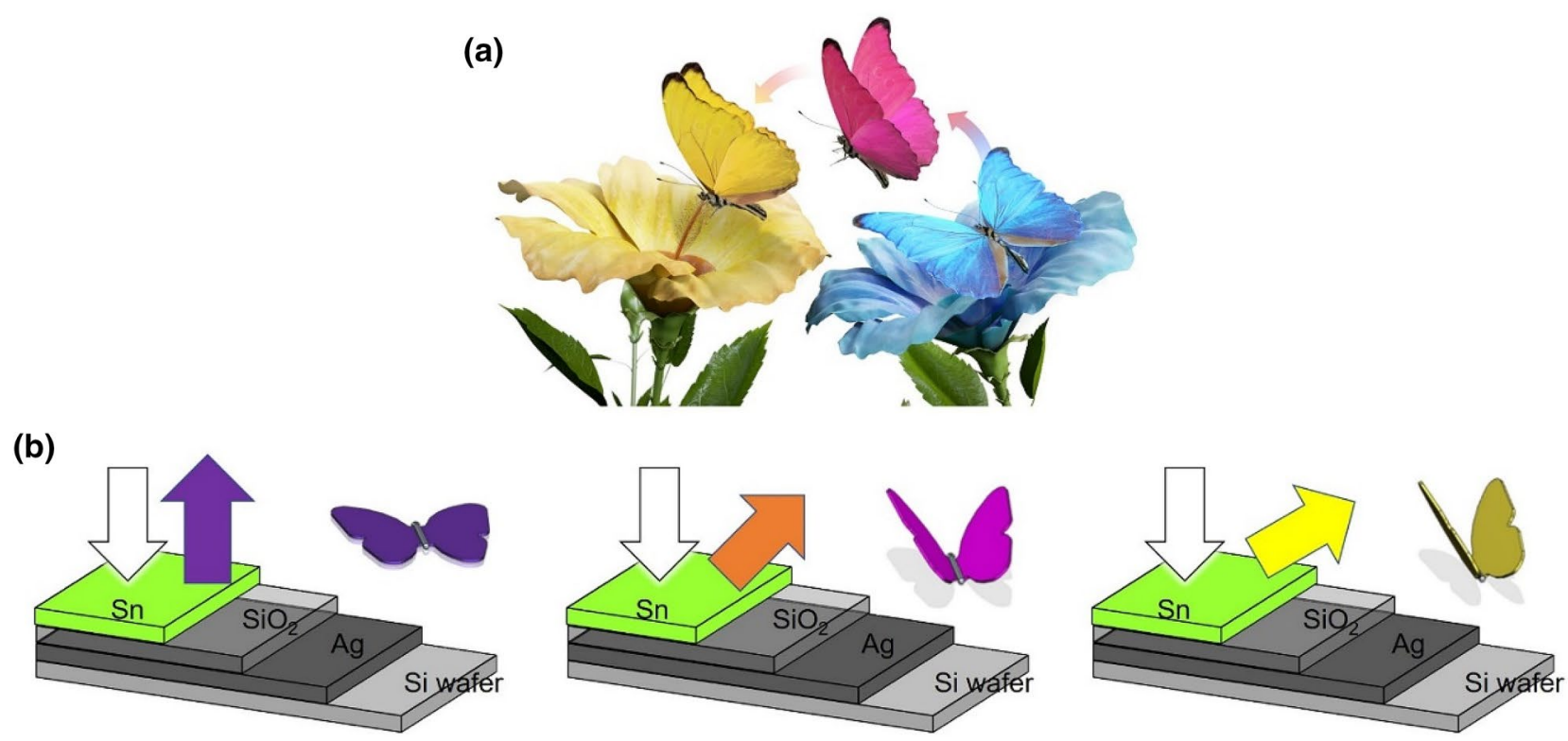

(c)
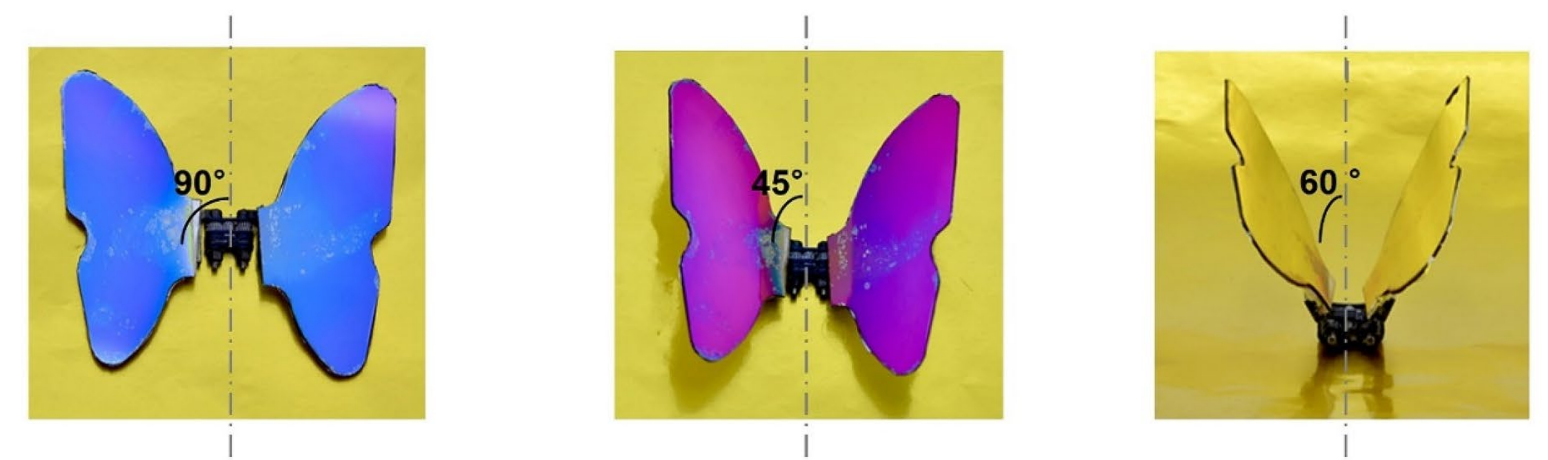

(d)
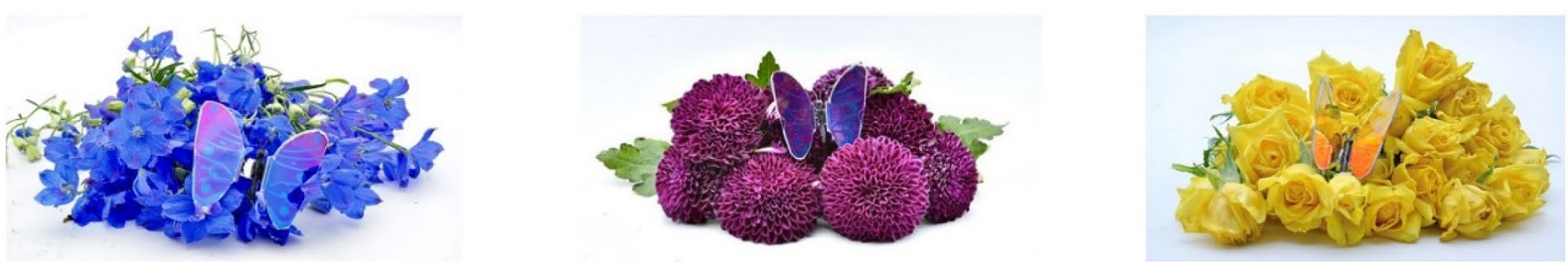

Fig. 1 Concept design of a pattern-free color-based angle sensor. a Camouflage of butterfly in nature (Rendering). b Color change by reflective angle in the Fabry-Perot (FP) using the metal-insulator-metal (MIM) structure composed of silver (Ag)-silicon dioxide

absorption band is too narrow. However, when the resonant absorption band is widened, the color change of the reflected light becomes easy to visually distinguish. To confirm this principle, MIM structures of $\mathrm{Ag}(100 \mathrm{~nm})$ with various thicknesses of $\mathrm{SiO}_{2}$ (range 100-440 nm, in 20-nm increments) were fabricated to obtain various structural colors (Figure S3, Supporting Information). The thickness of the Sn layer was determined to be $10 \mathrm{~nm}$, based on the simulation results shown in Fig. 2c. To verify the proposed principle, two samples, $\mathrm{Ag}-\mathrm{SiO}_{2}-\mathrm{Ag}$ and $\mathrm{Ag}-\mathrm{SiO}_{2}-\mathrm{Sn}$, were
$\left(\mathrm{SiO}_{2}\right)$-tin $(\mathrm{Sn})$; c A sample of butterfly robot with color of cyan, magenta and yellow according to the wing angles of $0^{\circ}, 45^{\circ}$, and $60^{\circ}$, respectively (Photo). d A camouflage of fabricated butterfly robot with respect to actual flower (Photo) (color figure online)

fabricated and their colorations were compared, as shown in Supporting Information Figures S2a, S2b.

To verify the angle sensitiveness of MIM structure, the thickness of the insulator, $\mathrm{SiO}_{2}$, was designed according to FDTD simulation results. As shown in Fig. 3, MIM structures with $\mathrm{SiO}_{2}$ thicknesses of 270 and 400 exhibited broad absorption peaks over the wavelength range of 400-700 nm, and the center wavelength of resonant absorption decreased with increasing angle. Additionally, the asymmetry of the MIM structure and the change in the refractive index 

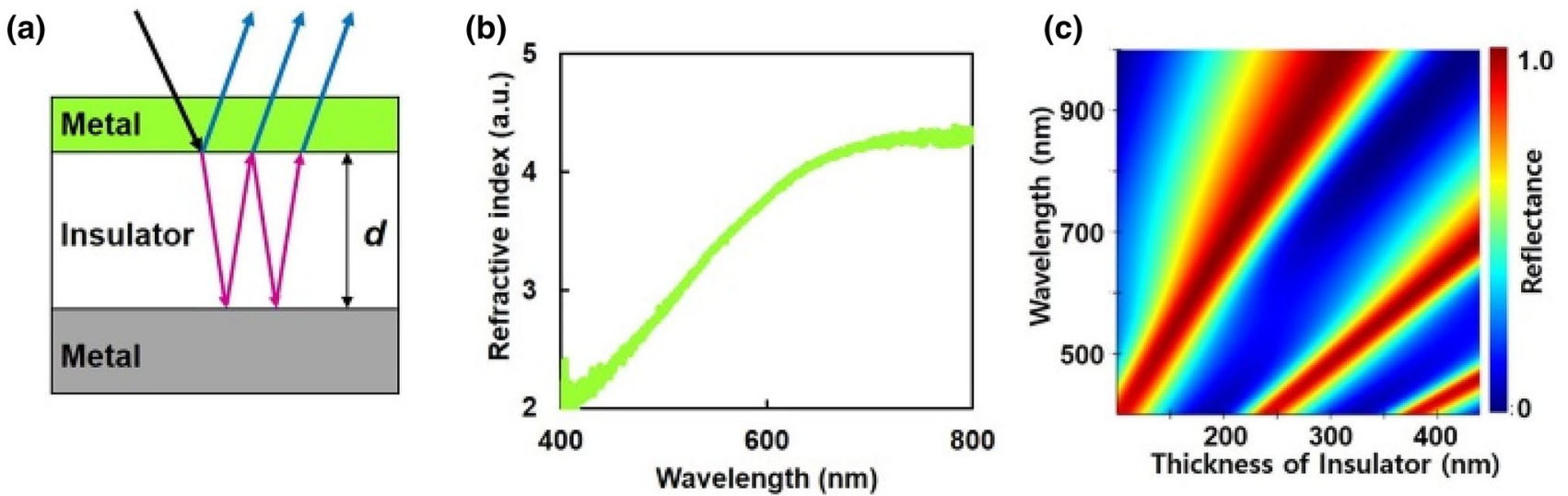

Fig. 2 Analysis of the MIM structure using the optical properties of $\mathrm{Sn}$. a FP reflectance of the MIM structure. $\mathbf{b}$ Refractive index of Sn according to the wavelength. The refractive index of Sn increases sig- nificantly for wavelengths in the visible regime. c Reflectance plotted as a function of wavelength and dielectric thickness using the refractive index of Sn and finite difference time domain (FDTD) analysis (a)

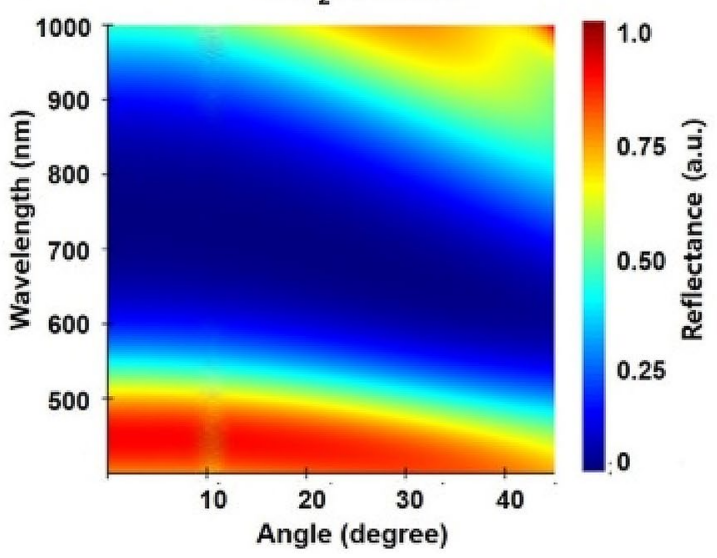

(b)

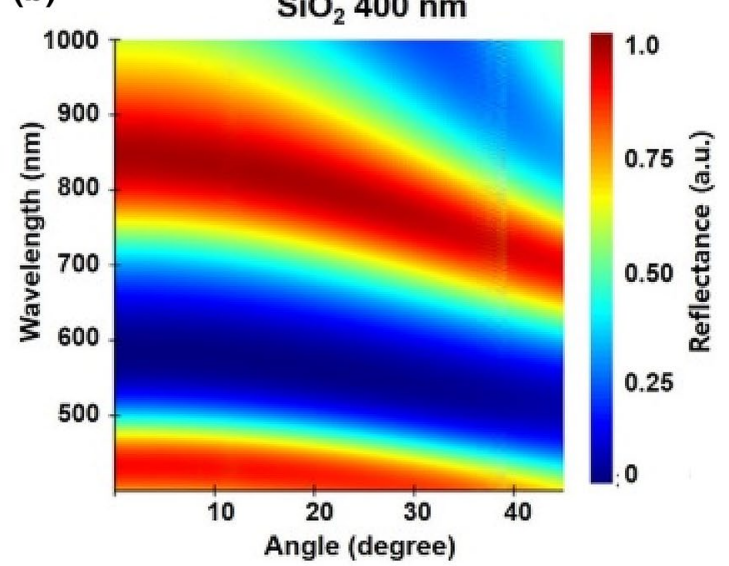

Fig. 3 FDTD analysis of the $\mathrm{Ag}_{-} \mathrm{SiO}_{2}-\mathrm{Sn} \mathrm{MIM}$ structure according to angle of a $\mathrm{Ag}(50 \mathrm{~nm})-\mathrm{SiO}_{2}(270 \mathrm{~nm})-\mathrm{Sn}(10 \mathrm{~nm})$, and b $\mathrm{Ag}(50 \mathrm{~nm})-$ $\mathrm{SiO}_{2}(400 \mathrm{~nm})-\mathrm{Sn}(10 \mathrm{~nm})$

according to the wavelength of Sn considerably increased the angle dependency of the reflected color. As shown in Supporting Information Figure S2c and S2d, the results of FDTD simulation of a symmetric MIM structure using $\mathrm{SiO}_{2}$ and $\mathrm{WO}_{3}$ as insulators are compared, but the change of the absorption pick with angle is smaller than that of the asymmetric structure MIM. Thus, the proposed MIM structure is suitable as an angle-detecting sensor, as the central wavelength of the structure decreased linearly for angles $\geq 10^{\circ}$.

Three designed MIM structures were fabricated with $\mathrm{SiO}_{2}$ thicknesses of 230, 270 and $400 \mathrm{~nm}$. The MIM structure having an insulating layer of 230 and $270 \mathrm{~nm}$ thickness has a single absorption peak, and the average value of the $\mathrm{q}$-factor is 2.83 . The reflected colors were detected under different reflecting angles from $0^{\circ}$ to $75^{\circ}$ in $15^{\circ}$-steps with a normal-incidence white light source (Figs. $4 \mathrm{a}$ and Supporting Information S5). The captured color images for all three samples exhibited visible color changes as the tilt angle was varied (Fig. 4b). We measured the thickness of the insulator layer using ellipsometry in Fig. 4c; samples fabricated with insulating layer thicknesses of 230, 270, and $400 \mathrm{~nm}$ had error values of $+4.07,+1.73$, and $-4.09 \%$, respectively, and the average error was $3.47 \%$. Figure $4 \mathrm{~d}$ presents a crosssectional scanning electron microscopy image of the sample with a $\mathrm{SiO}_{2}$ thickness of $230 \mathrm{~nm}$.

The reflected spectra of three samples with different $\mathrm{SiO}_{2}$ thicknesses under different angles were measured using spectrometry. We examined the color shift caused by angle tilting using four angles $\left(15^{\circ}, 30^{\circ}, 45^{\circ}\right.$, and $\left.60^{\circ}\right)$ for the three samples. The spectrum of absorbed light constantly shifted to the left by increasing the tilt angle, resulting in a change in the reflected color, as shown in Fig. 5a.

Figure $5 \mathrm{~b}$ presents changes in the central wavelength of resonant absorption as a function of angle; the center 
(a)

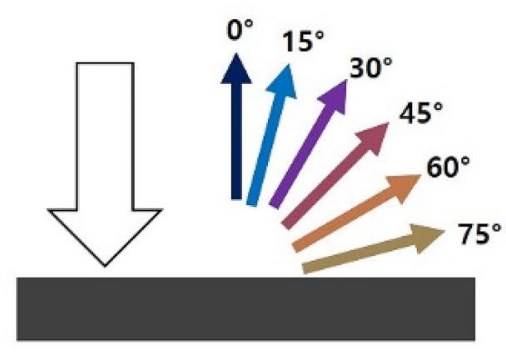

(b)

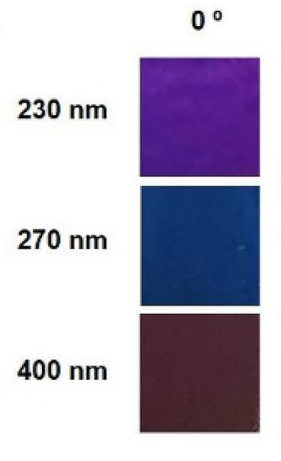

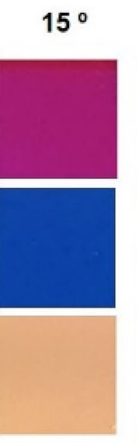

$30^{\circ}$

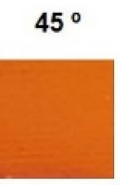

$60^{\circ}$

$75^{\circ}$
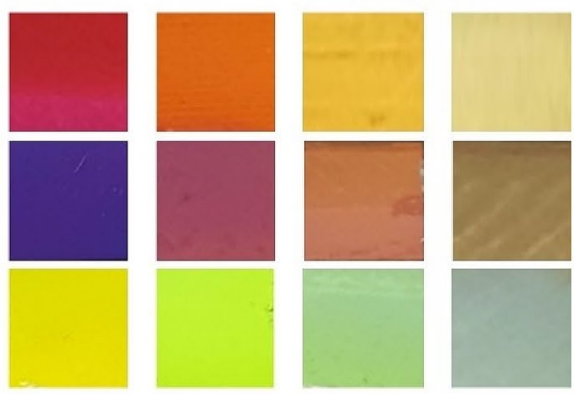

$+2$

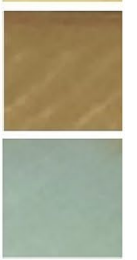

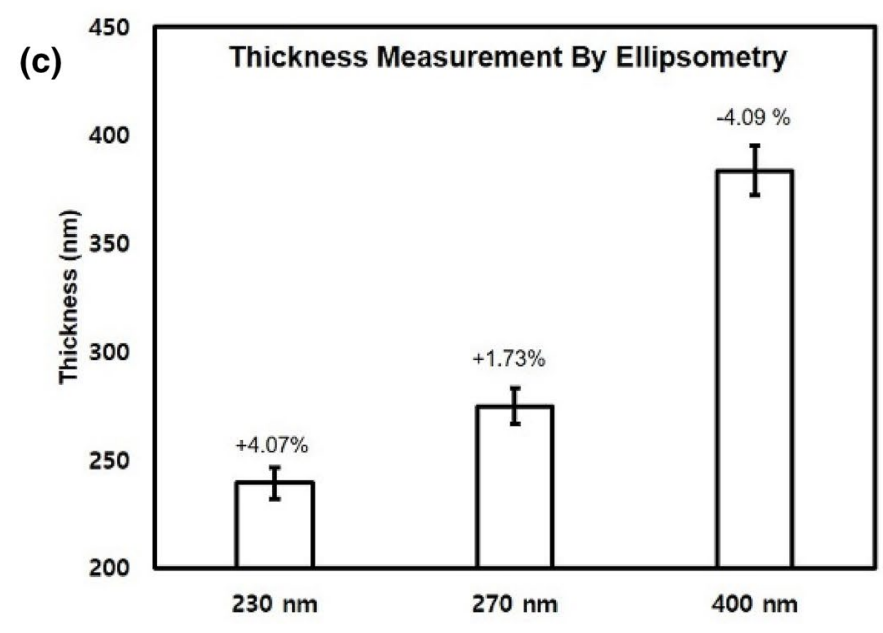

(d)

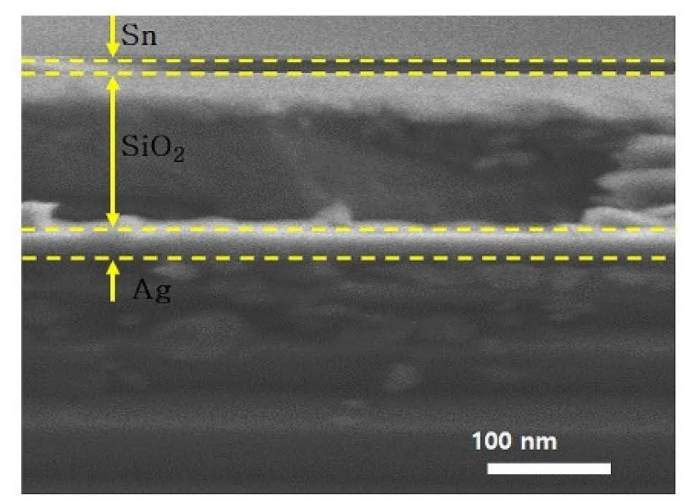

Fig. 4 Fabrication of a color-based angle sensor and characterization. a Concept of the angle-dependent color change sensor. b Color change from $0^{\circ}$ to $75^{\circ}$ for color-based angle sensors with a $\mathrm{SiO}_{2}$ thickness of 230, 270, and $400 \mathrm{~nm}$. c Comparison of the reflectance between the analytical value of an angle sensor with a $\mathrm{SiO}_{2}$ thickness

wavelengths of the three angle detection sensors shifted linearly. For a sensor with a $\mathrm{SiO}_{2}$ thickness of $230 \mathrm{~nm}$, the absorption peak of the central wavelength shifted from 573.1 to $490.1 \mathrm{~nm}$ as the angle changed from $15^{\circ}$ to $60^{\circ}$. This blue shift in the reflectance is consistent with FDTD simulation results, as evidenced by the change in color from blue to yellow (Fig. 4b). The measured value of a sensor with an insulating layer of $230 \mathrm{~nm}$ can be expressed as a linear function of the central wavelength shift $(\lambda)$ with respect to a change in the angle $(\theta)$.

$\lambda=1.81 \theta+\lambda_{0}$

Therefore, when using a spectrometer, the proposed structure realized an angle shifting of central wavelength of absorption with a sensitivity of 1.81 . To expand the designed MIM resonator system to angle-detecting sensor, we provide more simplified and intuitive measurement system. Figure $5 \mathrm{c}$ presents a monochromatic light method to measure the reflectance of the system; notably, a spectrometer is not required for this approach [36]. The reflectance at the of 230,270 , and $400 \mathrm{~nm}$ and the actual value measured by ellipsometry (error values of samples are $+4.07,+1.73$, and $-4.09 \%$, respectively, and the average error was $3.47 \%$ ). d Cross-sectional scanning electron microscopy image of the $\mathrm{Ag}(50 \mathrm{~nm})-\mathrm{SiO}_{2}(230 \mathrm{~nm})-\mathrm{Sn}$ $(10 \mathrm{~nm})$ MIM angle-detecting sensor (color figure online)

selected wavelength, as indicated by the vertical dashed line in Fig. 5a, was plotted as a function of angle in Fig. 5c. For the sensor with a 230-nm-thick insulating layer, the reflectance (R) using 620-nm monochromatic light is given by the linear function with respect to the angle $(\theta)$,

$R=1.71 \theta+R_{0}$

This consistency between full-spectrum data and reflectance at a specific wavelength indicates that the monochromatic light method is as effective as measuring the angle using a spectrometer. Figure $5 \mathrm{~d}$ presents color change according to the angle, as indicated by the MIM angledetecting sensor, using the CIE 1931 color coordinate system without an additional measuring device. The color spacing according to the angle of the color coordinate system is sufficiently wide; thus, the proposed angle-detecting sensor can project enough visual color change to be measured with the naked eye. Among the sensor prototypes tested, the angle sensor having the structure with a $\mathrm{SiO}_{2}$ thickness of $230 \mathrm{~nm}$ showed the largest change in distance in the coordinate 
(a)

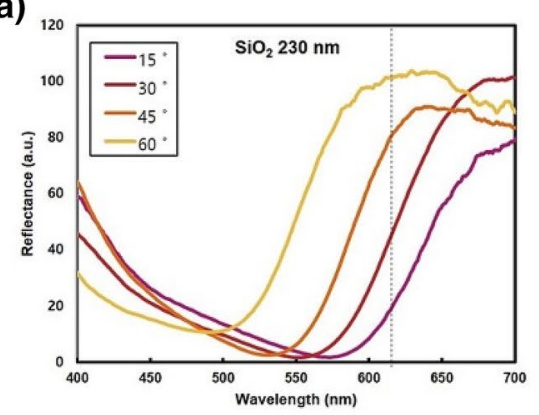

(b)

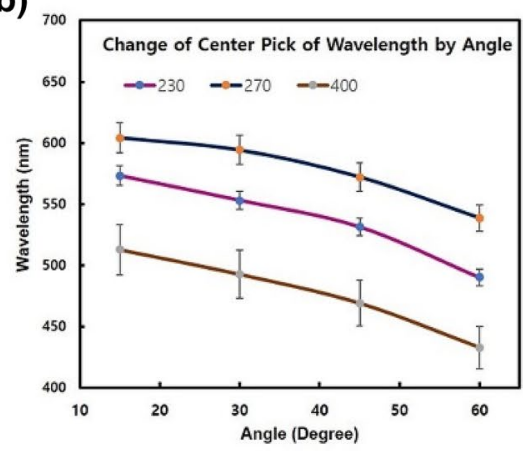

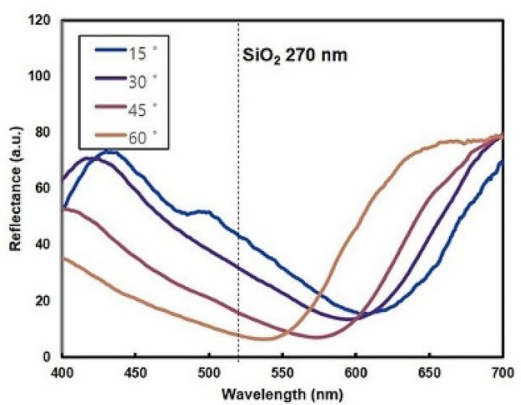

(c)

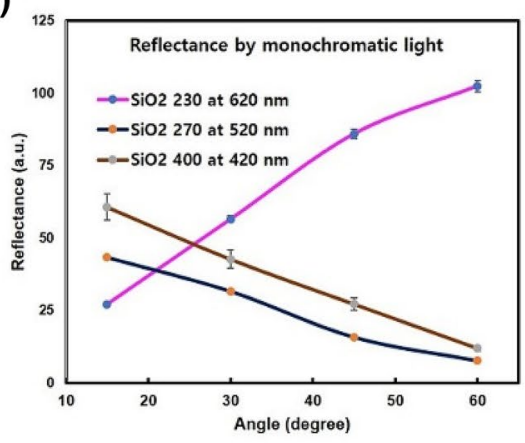

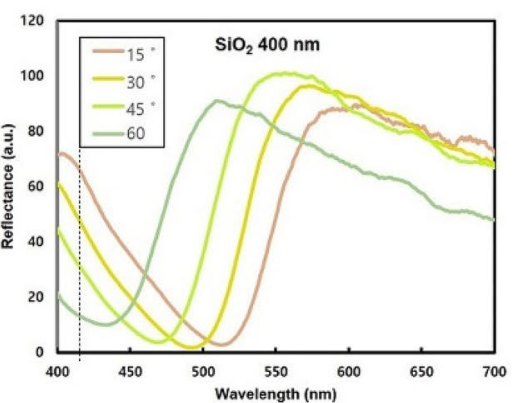

(d)

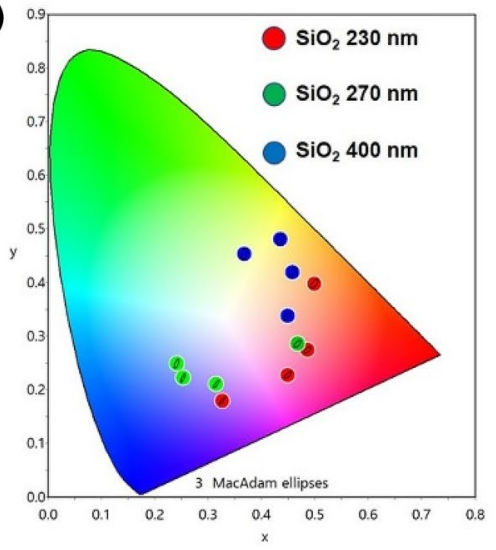

Fig. 5 Spectral analysis and characteristic analysis of angle sensor according to angle. a Spectroscopic analysis from $15^{\circ}$ to $60^{\circ}$ for a color-based angle sensor with a $\mathrm{SiO}_{2}$ thickness of 230, 270, and $400 \mathrm{~nm}$. b Change in the central wavelength of the angle sensor according to the angle of each $\mathrm{SiO}_{2}$ thickness. c Reflectance value

system, so it is the most sensitive color sensor for visual assessments.

\section{Application}

Due to the demand for product design competitiveness, 3D curved designs are gaining more attention in industries, such as in mobile manufacturing. Most components of electronic products with 3D curved surfaces are manufactured using transparent materials, such as glass, ceramics, and hard-coated optical polymers. The transparent items contain highly glossy parts with a 3D curved surface, making it difficult to detect the shape quality of the curved surfaces. Furthermore, current inspection processes for 3D curved shapes require vision-based detecting devices, such as multiple charge-coupled devices (CCDs) or 3D laser scanners with high resolution, which complicates system integration. To simplify the detecting method and minimize costs, the proposed color-based angle-detecting sensor can be applied.

To demonstrate our sensor prototype, we applied our sensor to a 3D curved back cover of a mobile phone. The sensor was made of a flexible polyethylene terephthalate film of the angle sensor measured at a specific monochromatic wavelength band $\left(230-\mathrm{nm} \mathrm{SiO}_{2}\right.$ sample at $620, \mathrm{SiO}_{2} 270 \mathrm{~nm}$ sample at $520, \mathrm{SiO}_{2}$ $400 \mathrm{~nm}$ sample at 420). d CIE 1931 color coordinate display of the color shift of the angle sensor with three thicknesses of $\mathrm{SiO}_{2}$ (color figure online)

with a thickness of $100 \mathrm{um}$ and the adhesive was a silicone pressure sensitive adhesive with a thickness of $30 \mathrm{um}$. The MIM structure was fabricated on the substrate, and applied to a curved surface inspection with a minimum curvature of 2R. Figure 6a presents sections of the 3D curved back cover of a mobile phone and the shape difference between the designed shape and failure samples in section A-A'. The sensor prototype, fabricated with a 230 -nm-thick $\mathrm{SiO}_{2}$ layer, was attached to the back cover, as shown in Fig. 6b. The color change of the sensor was observed visually over the curved surface of the phone. We used a 3D laser scanning method to verify the results obtained with our sensor. Figure $6 \mathrm{c}$ presents the difference in slope measured by laser scanning between the 'pass' and 'fail' samples; a large color difference was observed at point $b$ compared with point $c$, as shown in Fig. 6b. We checked the color difference using spectroscopic analysis, as shown in Fig. 6d; an error of $5.45 \%$ was obtained as shown in Fig. 6e, due to difficulty in aligning the position of the 3D laser for scanning and spectrometry. However, these results indicate that quality inspection of the 3D curved surface is possible by visually identifying color without 3D scanning or inspection with a CCD. Furthermore, using the color map and a spectrometer 
(a)

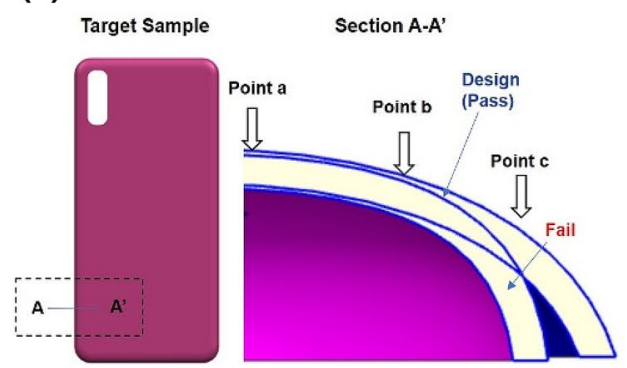

(d)

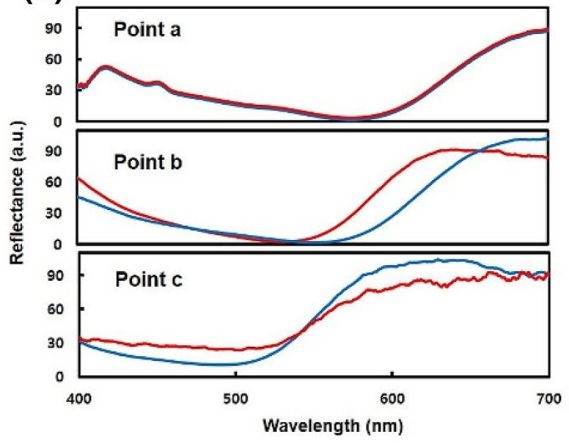

Fig. 6 3D shape detection test results. a Section of the tested sample (back cover of a mobile phone). b The 3D shape detector on the $3 \mathrm{D}$ curved back cover edge and difference in color change of sensor for passed and failed samples. c The measured 3D shape of the back cover via a laser scanning method. $\mathbf{d}$ Spectroscopic analysis of three

in parallel enables better quality control while simplifying the inspection process.

We propose a new inspection process based on 3D shape detection technology (Supporting Information Figure S6). If the $3 \mathrm{D}$ part is made by thermoforming, then a protective MIM coating can be added to the part. This enables inspection of the 3D curved surface with the naked eye, either by taking a picture with a camera or using a spectrometer. This technology also enables automation of the manufacturing and inspection processes. Hence, we can significantly improve the inspection efficiency in situations where it is difficult to apply conventional inspection methods. Furthermore, real-time 3D shape detection using a color-changing film may offer a means for rapid diagnosis of molding problems and a reduction in product development costs for low-temperature $\left(<200{ }^{\circ} \mathrm{C}\right)$ molding, such as cold press processes.

\section{Conclusion}

In this study, we developed a wireless color-based angle sensor based on a FP interferometer design that does not require nano-patterning processes. To increase the

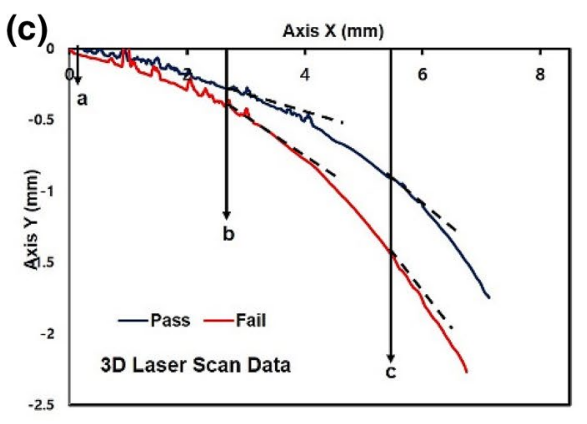

(e)

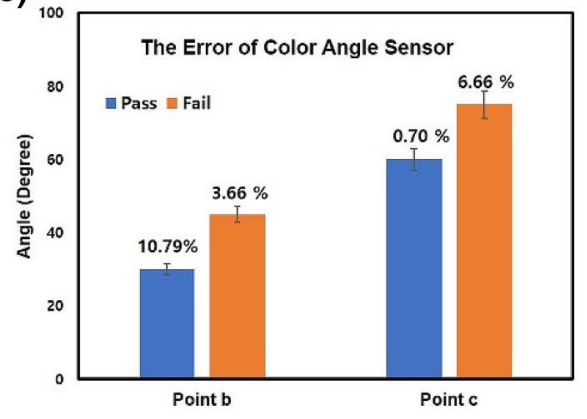

points. e Comparison of measurement values between the laser scan and color angle sensor. The cause of the error is the instability of the laser measurement curve and the measurement error according to the angle of the spectrometer (color figure online)

reflected color change according to the angle, we propose a color element using an asymmetric MIM structure, with $\mathrm{Sn}$ and $\mathrm{Ag}$ as the metal layers. The light absorption properties of Sn create a MIM structure with a wide absorption bandwidth in the visible light region, and through this, vivid colors and a full spectrum are realized, even with reflected light. Additionally, the asymmetry of the MIM structure and the change in the refractive index according to the wavelength of Sn considerably increases the angle dependency of the reflected color. This valuable feature can be used in practical industrial applications involving high throughput for a low cost.

Our proposed sensor is expected to be useful in measurement and inspection technologies, such as those required for shape detection of 3D curved transparent parts of electronic devices [37-39]. It can measure the quality of $3 \mathrm{D}$ curved surfaces without the need for additional measuring equipment and does not require a separate scanning time or calculation time. In conclusion, our proposed sensor is simple in its design and enables rapid measurement for use in industrial applications. 


\section{Methods}

\subsection{Metal Deposition}

Metal deposition was performed using an EB-PVD (SamHan Thin Film \& Vacuum). To obtain smooth, highquality films, the pressure of the evaporation chamber was adjusted to $9 \times 10-7$ Torr by pre-pumping. Meanwhile, the working pressure was maintained below $1 \times 10-6$ Torr. A 50-nm-thick layer of Ag was deposited on top of a polycarbonate-polymethyl methacrylate composite substrate at a rate of $1.5-2.0 \AA \mathrm{s}-1$. Next, a 100-440-nm-thick layer of $\mathrm{SiO}_{2}$ was deposited onto the developed samples at a deposition rate of 1.5-2.0 $\mathrm{A} s-1$, followed by a $10-\mathrm{nm}$-thick layer of $\mathrm{Sn}$ at a deposition rate of 0.4-0.5 $\mathrm{s}-1$. The temperature of the sample chamber was maintained below $25{ }^{\circ} \mathrm{C}$ during the evaporation process. The film thickness was monitored using a quartz crystal microbalance, with angstrom-level sensitivity.

\subsection{Morphology and Optical Characterization}

Microscopy analysis was performed using an Olympus BX53M instrument (Olympus Corp., Tokyo, Japan). The eyepiece used was a U-TV0.5XC-3 (Olympus); the objective lens was an LMPlan FL N 10X/0.30, FN26.5, and the CCD camera was an MS300. The software used for image capture was Leopard v2.0 lite.

Spectroscopic analysis was performed using a miniature spectrometer (QP400-2-VIS-NIR; Ocean Optics/Ocean Insight). As a reference, we used standard white for the Scinco Colormate spectrophotometer (Scinco, Daejeon, Korea). We carried out the spectral analysis using Ocean View software (version 1.6.3) and an FDTD solution program (version 8.21.1781) for preliminary spectral analysis. Mapping software Color Calculator (version 7.59; OsRam Licht AG, Munich, Germany) was used in conjunction with the CIE 1931 standard.

The refractive index of Sn was measured with a dualrotating compensator spectroscopic ellipsometer; a halogen lamp was used as the light source. A Maya 2000 Pro spectrometer was used for the ellipsometry measurements (Ocean Optics/Ocean Insight) and the objective lens was an Olympus SLMPlan N $50 \times$ objective (numerical aperture, 0.35).

Supplementary Information The online version contains supplementary material available at https://doi.org/10.1007/s40684-021-00324-9.

Acknowledgements This work was supported by the National Research Foundation of Korea (NRF) grant funded by the Korea Government (MSIT) (No. NRF-2018R1A2A1A13078704), the Basic Research Lab Program (No. NRF 2018R1A4A1059976), Institute of Engineering Research, Seoul National University (SNU). Ellipsometry measurements were conducted to determine film thickness, with the help of the Thin Film Process Laboratory of Heui-Jae Pakh, SeungWoo Lee, Seoul National University, South Korea.

\section{Compliance with Ethical Standards}

Conflict of Interest On behalf of all authors, the corresponding author states that there is no conflict of interest.

Open Access This article is licensed under a Creative Commons Attribution 4.0 International License, which permits use, sharing, adaptation, distribution and reproduction in any medium or format, as long as you give appropriate credit to the original author(s) and the source, provide a link to the Creative Commons licence, and indicate if changes were made. The images or other third party material in this article are included in the article's Creative Commons licence, unless indicated otherwise in a credit line to the material. If material is not included in the article's Creative Commons licence and your intended use is not permitted by statutory regulation or exceeds the permitted use, you will need to obtain permission directly from the copyright holder. To view a copy of this licence, visit http://creativecommons.org/licenses/by/4.0/.

\section{References}

1. Duan, X., Kamin, S., \& Liu, N. (2017). Dynamic plasmonic colour display. Nature Communications, 8, 1-9.

2. Franklin, D., Frank, R., Wu, S.-T., \& Chanda, D. (2017). Actively addressed single pixel full-colour plasmonic display. Nature Communications, 8, 1-10.

3. Quan, Y. J., Kim, Y. G., Kim, M. S., Min, S.-H., \& Ahn, S.-H. (2020). Stretchable bi-axial and shear strain sensors using diffractive structural colors. ACS Nano, 14, 5392-5399.

4. Quan, Y. J., Kim, M. S., Kim, Y., \& Ahn, S.-H. (2019). Colourtunable $50 \%$ strain sensor using surface-nanopatterning of soft materials via nanoimprinting with focused ion beam milling process. CIRP Annals, 68, 595-598.

5. Sandt, J. D., Moudio, M., Clark, J. K., Hardin, J., Argenti, C., Carty, M., \& Lewis, J. A. (2018). Kolle, Stretchable optomechanical fiber sensors for pressure determination in compressive medical textiles. Advanced Healthcare Materials, 7, 1800293.

6. Wu, S., Liu, T., Tang, B., Li, L., \& Zhang, S. (2019). Interfaces, structural color circulation in a bilayer photonic crystal by increasing the incident angle. ACS Applied Materials \& Interfaces, 11, 10171-10177.

7. Yakovlev, A. V., Milichko, V. A., Vinogradov, V. V., \& Vinogradov, A. V. (2016). Inkjet color printing by interference nanostructures. ACS Nano, 10, 3078-3086.

8. Yang, Z., Chen, Y., Zhou, Y., Wang, Y., Dai, P., Zhu, X., \& Duan, H. (2017). Microscopic interference full-color printing using grayscale-patterned fabry-perot resonance cavities. Advanced Optical Materials, 5, 1700029.

9. Kristensen, A., Yang, J. K., Bozhevolnyi, S. I., Link, S., Nordlander, P., Halas, N. J., \& Mortensen, N. (2016). Plasmonic colour generation. Nature Reviews Materials, 2, 1-14.

10. Han, M. G., Shin, C. G., Jeon, S. J., Shim, H., Heo, C. J., Jin, H., et al. (2012). Full color tunable photonic crystal from crystalline colloidal arrays with an engineered photonic stop-band. Advanced Materials, 24, 6438-6444.

11. Guo, D.-Y., Chen, C.-W., Li, C.-C., Jau, H.-C., Lin, K.-H., Feng, T.-M., et al. (2020). Reconfiguration of three-dimensional liquidcrystalline photonic crystals by electrostriction. Nature Materials, 19, 94-101. 
12. Dao, T. D., Chen, K., Ishii, S., Ohi, A., Nabatame, T., Kitajima, M., \& Nagao, T. (2015). Infrared perfect absorbers fabricated by

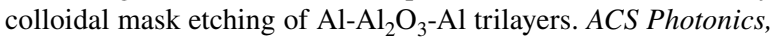
2, 964-970.

13. Li, Z., Butun, S., \& Aydin, K. (2015). Large-area, lithographyfree super absorbers and color filters at visible frequencies using ultrathin metallic films. ACS Photonics, 2, 183-188.

14. Dai, P., Wang, Y., Zhu, X., Shi, H., Chen, Y., Zhang, S., et al. (2018). Transmissive structural color filters using vertically coupled aluminum nanohole/nanodisk array with a triangular-lattice. Nanotechnology, 29, 395202.

15. Hong, J., Chan, E., Chang, T., Fung, T. C., Hong, B., Kim, C., et al. (2015). Continuous color reflective displays using interferometric absorption. Optica, 2, 589-597.

16. Wang, H., Wang, X., Yan, C., Zhao, H., Zhang, J., Santschi, C., \& Martin, O. J. (2017). Full color generation using silver tandem nanodisks. ACS Nano, 11, 4419-4427.

17. Yang, Z., Zhou, Y., Chen, Y., Wang, Y., Dai, P., Zhang, Z., \& Duan, H. (2016). Reflective color filters and monolithic color printing based on asymmetric Fabry-Perot cavities using nickel as a broadband absorber. Advanced Optical Materials, 4, 1196-1202.

18. Kats, M. A., Blanchard, R., Genevet, P., \& Capasso, F. (2013). Nanometre optical coatings based on strong interference effects in highly absorbing media. Nature Materials, 12, 20-24.

19. Lee, K. T., Han, S. Y., \& Park, H. J. (2017). Omnidirectional flexible transmissive structural colors with high-color-purity and high-efficiency exploiting multicavity resonances. Advanced Optical Materials, 5, 1700284.

20. Park, S. I., Quan, Y. J., Kim, S. H., Kim, H., Kim, S., Chun, D.-M., et al. (2016). A review on fabrication processes for electrochromic devices. International Journal of Precision Engineering and Manufacturing - Green Technology, 3, 397-421.

21. Cu-Nguyen, P. H., Grewe, A., Feßer, P., Seifert, A., Sinzinger, S., \& Zappe, H. (2016). An imaging spectrometer employing tunable hyperchromatic microlenses. Light: Science and Applications, 5, e16058.

22. Lee, J. Y., Lee, K. T., Seo, S., \& Guo, L. J. (2014). Decorative power generating panels creating angle insensitive transmissive colors. Scientific Reports, 4, 4192.

23. Yang, C., Shen, W., Zhang, Y., Li, K., Fang, X., Zhang, X., \& Liu, X. (2015). Compact multilayer film structure for angle insensitive color filtering. Scientific Reports, 5, 9285.

24. Chen, Y., Duan, X., Matuschek, M., Zhou, Y., Neubrech, F., Duan, H., \& Liu, N. (2017). Dynamic color displays using stepwise cavity resonators. Nano Letters, $17,5555-5560$.

25. Lee, K.-T., Lee, J. Y., Seo, S., \& Guo, L. J. (2014). Colored ultrathin hybrid photovoltaics with high quantum efficiency. Light: Science and Applications, 3, e215.

26. Mirshafieyan, S. S., Guo, H., \& Guo, J. (2016). Zeroth order fabryperot resonance enabled strong light absorption in ultrathin silicon films on different metals and its application for color filters. IEEE Photonics, 8, 1-12.

27. Mirshafieyan, S. S., \& Guo, J. (2014). Silicon colors: spectral selective perfect light absorption in single layer silicon films on aluminum surface and its thermal tunability. Optics Express, 22, 31545-31554.

28. Lee, K. T., Seo, S., Lee, J. Y., \& Guo, L. J. (2014). Strong resonance effect in a lossy medium-based optical cavity for angle robust spectrum filters. Advanced Materials, 26, 6324-6328.

29. Kats, M. A., Byrnes, S. J., Blanchard, R., Kolle, M., Genevet, P., Aizenberg, J., \& Capasso, F. (2013). Enhancement of absorption and color contrast in ultra-thin highly absorbing optical coatings. Applied Physics Letters, 103, 101104.

30. Kwon, H., \& Kim, S. (2015). Chemically tunable, biocompatible, and cost-effective metal-insulator-metal resonators using silk protein and ultrathin silver films. ACS Photonics, 2, 1675-1680.

31. Kim, T., Yu, E. S., Bae, Y. G., Lee, J., Kim, I. S., Chung, S., et al. (2020). Asymmetric optical camouflage: tuneable reflective colour accompanied by the optical Janus effect. Light: Science \& Applications, 9(1), 1-10.

32. Cho, S. M., Cheon, S. H., Kim, T.-Y., Ah, C. S., Song, J., Ryu, H., \& Chu, H. Y. (2016). Design and fabrication of integrated fabry-perot type color reflector for reflective displays. Journal of Nanoscience and Nanotechnology, 16, 5038-5043.

33. Zhang, Y., Duan, J., Wu, M., Zhi, Y., Li, X., Zhou, Y., et al. (2018). Integrated structural color array enabled by ultrathin silver film via cavity-enhanced absorption. Optical and Quantum Electronics, 50, 161.

34. Kawaguchi, H., Okita, T., Tamaki, H. (1986). Antistatic plastic films. Google Patents US Patent 4,571,361

35. Lee, K. T., Seo, S., \& Guo, L. J. (2015). High-color-purity subtractive color filters with a wide viewing angle based on plasmonic perfect absorbers. Advanced Optical Materials, 3, 347-352.

36. Snapp, P., Kang, P., Leem, J., \& Nam, S. J. (2019). Colloidal photonic crystal strain sensor integrated with deformable graphene phototransducer. Advanced Functional Materials, 29, 1902216.

37. Lu, W., Li, H., Huo, B., Meng, Z., Xue, M., Qiu, L., et al. (2016). Full-color mechanical sensor based on elastic nanocomposite hydrogels encapsulated three-dimensional colloidal arrays. Sensors and Actuators B: Chemical, 234, 527-533.

38. Wang, F., Zhu, Z., Xue, M., Xue, F., Wang, Q., Meng, Z., et al. (2015). Cellulose photonic crystal film sensor for alcohols. Sensors and Actuators B: Chemical, 220, 222-226.

39. Zhang, Z., Wang, Y., Huang, S., Liu, Y., Chang, C., Gao, F., \& Jiang, X. (2017). Three-dimensional shape measurements of specular objects using phase-measuring deflectometry. Sensors, 17, 2835.

Publisher's Note Springer Nature remains neutral with regard to jurisdictional claims in published maps and institutional affiliations.

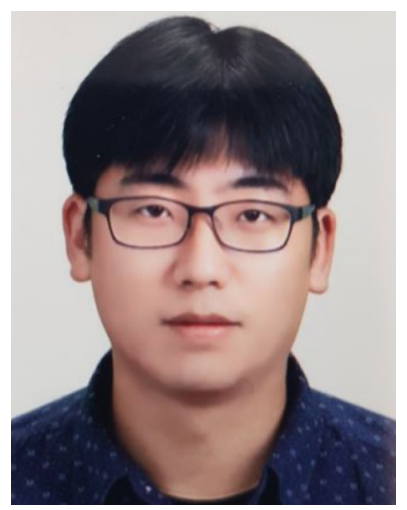

Young-Gyun Kim has worked for Samsung Electronics since 2005. $\mathrm{He}$ received an MS degree in mechanical engineering from Yonsei University in 2014. He is currently a Ph.D. Candidate at Seoul National University. His research interests include structural color sensor, flexible smart materials and smart manufacturing system.

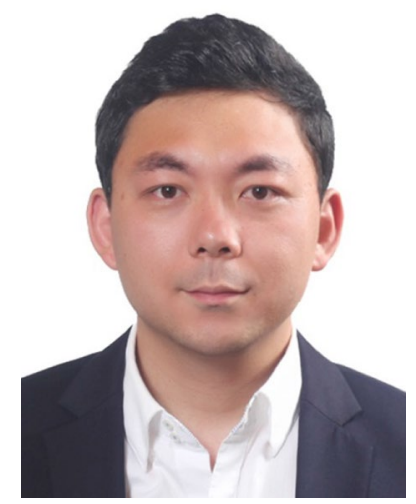

Ying-Jun Quan received a Ph.D. degree in mechanical and aerospace engineering from Seoul National University in 2019. He is currently a postdoctoral researcher in Institute of Advanced Machines and Design at Seoul National University. His research interests include micro/ nanoscale fabrication, structural color sensor, and smart manufacturing system. 


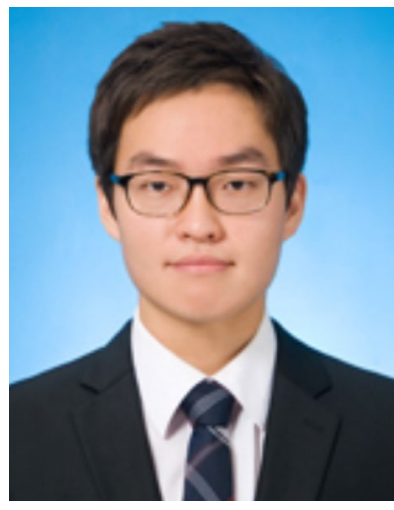

Min-Soo Kim received a Ph.D. degree in mechanical engineering from Seoul National University in 2018 . He is currently a postdoctoral associate at ETH Zurich. His research interests include micro-/nanoscale fabrication, smart materials, and microrobotics.

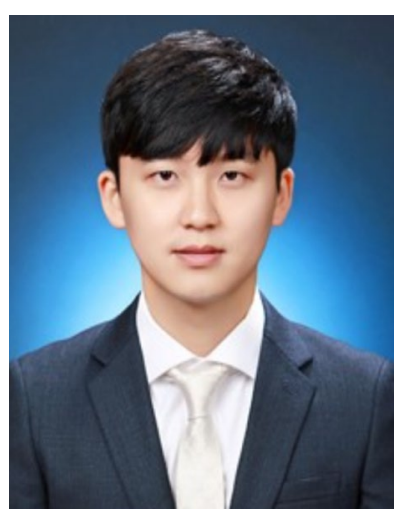

Younggyun Cho received an MS degree in mechanical and aerospace engineering from Seoul National University in 2021. He is currently a researcher in Global Technology Center at Samsung Electronics. His research interests include micro/ nanoscale fabrication, structural color sensor, and smart manufacturing system.

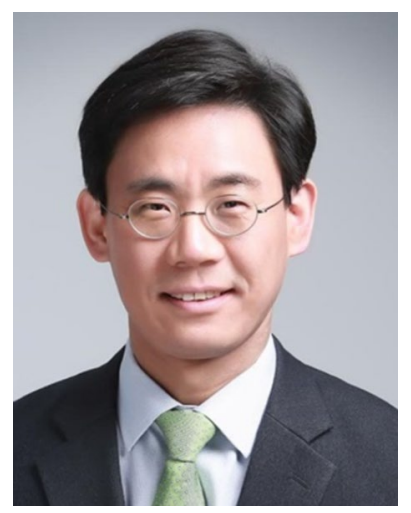

Sung-Hoon Ahn received a Ph.D. degree from Stanford University in 1997. He has been affiliated with UC Berkeley, Gyeongsang National University, University of Washington, and Hyundai WIA Corp. He is currently a full professor in the department of mechanical engineering at Seoul National University. His research interests include smart factory, green manufacturing, soft robotics, 3D printing, composite materials, micro/nano fabrications, renewable energy, and appropriate technology. 